\title{
Positivity of Schur function expansions of Thom polynomials
}

\author{
by \\ Piotr Pragacz and Andrzej Weber (Warszawa)
}

\begin{abstract}
Combining the approach to Thom polynomials via classifying spaces of singularities with the Fulton-Lazarsfeld theory of cone classes and positive polynomials for ample vector bundles, we show that the coefficients of the Schur function expansions of the Thom polynomials of stable singularities are nonnegative with positive sum.
\end{abstract}

1. Introduction. The global behavior of singularities $\left({ }^{1}\right)$ is governed by their Thom polynomials (cf. [17], [2], [9], [16]). As these polynomials are quite complicated even for "simplest" singularities, it is important to study their structure. There is a recent attempt to present Thom polynomials via their Schur function expansions (cf. [5], [13], [14]) instead of using the "traditional" basis of monomials in Chern classes.

In the present paper, we study the Schur function expansions of Thom polynomials from a "qualitative" point of view. In contrast to [16], [13], [14], where the Szücs-Rimányi approach via symmetries of singularities was used, we follow here the Kazarian approach [9] to Thom polynomials. In fact, both approaches rely on suitable "classifying spaces of singularities". We substitute the jet automorphism group by the group of the linear transformations $G L_{m} \times G L_{n}$. This allows one to extend the definition of Thom polynomials for maps $f: M \rightarrow N$ of complex manifolds to pairs of vector bundles. It is convenient to pass to homotopy theory, where each pair of bundles can be pulled back from the universal pair of bundles on $B G L_{m} \times$ $B G L_{n}$.

2000 Mathematics Subject Classification: 05E05, 14C17, 14N15, 55R40, 57R45.

Key words and phrases: Thom polynomials, classifying spaces of singularities, Schur functions, ample vector bundles, numerical positivity.

Research of P. Pragacz supported by a KBN grant and by the Humboldt Stiftung during the stay at the MPIM in Bonn.

Research of A. Weber supported by the KBN grant 1 P03A 00526.

$\left({ }^{1}\right)$ In the present paper we study complex singularities. 
We apply the Fulton-Lazarsfeld theory of cone classes and positive polynomials for ample vector bundles [7], and deduce nonnegativity of the coefficients in the Schur function expansions of the Thom polynomials of the singularities stable under suspension, with positive sum.

This "positivity" was previously checked for a number of singularities: by Thom [17] for $A_{1}(r)$, by Fehér and Kömüves [5] for some second order Thom-Boardman singularities, by the first author [13], [14] for $I_{2,2}(r), A_{3}(r)$, and for $A_{i}(r)$ (for all $i$ ) under the aditional assumption that $\Sigma^{j}(f)=\emptyset$ for $j \geq 2$, by the first author and Öztürk for Thom polynomials from [16], by the second author for the Thom polynomials (from [9]) of singularities of functions, and by Öztürk [12] for $A_{4}(3), A_{4}(4)\left({ }^{2}\right)$. Some of these examples are listed in the last section.

The paper is a revised version of the MPIM Bonn Preprint 2006-60.

2. Thom polynomials. Fix $m, n, k \in \mathbb{N}$. We denote by Aut ${ }_{n}$ the group of $k$-jets of automorphisms of $\left(\mathbb{C}^{n}, 0\right)$, and by $\mathcal{J}=\mathcal{J}(m, n)$ the space of $k$-jets of functions $\left(\mathbb{C}^{m}, 0\right) \rightarrow\left(\mathbb{C}^{n}, 0\right)\left({ }^{3}\right)$.

Moreover, we set

$$
G=\operatorname{Aut}_{m} \times \operatorname{Aut}_{n} .
$$

Consider the classifying principal $G$-bundle $E G \rightarrow B G$, i.e. a contractible space $E G$ with a free action of the group $G$, and define

$$
\widetilde{\mathcal{J}}=\widetilde{\mathcal{J}}(m, n)=E G \times_{G} \mathcal{J} .
$$

Let $\Sigma \subset \mathcal{J}$ be an analytic closed $G$-invariant subset, which we shall call a "class of singularities". For a given class of singularities $\Sigma$, set

$$
\widetilde{\Sigma}=E G \times_{G} \Sigma \subset \widetilde{\mathcal{J}}
$$

and denote by $\mathcal{T}^{\Sigma} \in H^{2 \operatorname{codim}(\Sigma)}(\widetilde{\mathcal{J}}, \mathbb{Z})$ the dual class of $[\widetilde{\Sigma}]\left({ }^{4}\right)$. Since

$$
H^{\bullet}(\tilde{\mathcal{J}}, \mathbb{Z}) \cong H^{\bullet}(B G, \mathbb{Z}) \cong H^{\bullet}\left(B G L_{m} \times B G L_{n}, \mathbb{Z}\right),
$$

$\mathcal{T}^{\Sigma}$ is identified with a polynomial in $c_{1}, \ldots, c_{m}$ and $c_{1}^{\prime}, \ldots, c_{n}^{\prime}$, the Chern classes of universal bundles $R_{m}$ and $R_{n}$ on $B G L_{m}$ and $B G L_{n}$ :

$$
\mathcal{T}^{\Sigma}=\mathcal{T}^{\Sigma}\left(c_{1}, \ldots, c_{m}, c_{1}^{\prime}, \ldots, c_{n}^{\prime}\right)
$$

$\left({ }^{2}\right)$ We use here the notation from [13], [14]. The calculations in the last three cases extensively used ACE [19].

$\left({ }^{3}\right)$ Though these objects depend on $k$, we omit " $k$ " in the notation. This also concerns other objects introduced later.

$\left({ }^{4}\right)$ One may approximate $E G \rightarrow B G$ by a sequence of $G$-bundles over finitedimensional manifolds $(E G)_{N} \rightarrow(B G)_{N}$, where $N \rightarrow \infty$. Then " $\mathcal{T}^{\Sigma}$ is the dual class of $[\widetilde{\Sigma}]$ " means that for any $N$ the image of $\mathcal{T}^{\Sigma}$ in $H^{\bullet}\left((B G)_{N}, \mathbb{Z}\right) \cong H^{\bullet}\left((E G)_{N} \times_{G} \mathcal{J}, \mathbb{Z}\right)$ is Poincaré dual to the class $\left[(E G)_{N} \times_{G} \Sigma\right]$ in $H_{\bullet}^{B M}\left((E G)_{N} \times_{G} \mathcal{J}, \mathbb{Z}\right)$. 
(here, and in the following, we omit the pull back in the notation). This is a classical Thom polynomial. Speaking slightly informally, given a general map $f: M \rightarrow N$ of smooth varieties of respective dimensions $m$ and $n$, the Thom polynomial

$$
\mathcal{T}^{\Sigma}\left(c_{1}(M), \ldots, c_{m}(M), c_{1}(N), \ldots, c_{n}(N)\right)
$$

gives the value of the dual class of the set where $f$ has singularity "of the class $\Sigma "$. A precise version of this statement is a content of the Thom theorem [17] (see also [9, Theorem 1] and [16, Sect. 6]).

The suspension

$$
\mathcal{S}: \mathcal{J}(m, n) \hookrightarrow \mathcal{J}(m+1, n+1)
$$

allows one to increase the dimension of the source and target simultaneously: with the local coordinates $x_{1}, x_{2}, \ldots$ for the source and a function $f=$ $f\left(x_{1}, \ldots, x_{m}\right)$, the jet $(\mathcal{S} f) \in \mathcal{J}(m+1, n+1)$ is defined by

$$
(\mathcal{S} f)\left(x_{1}, \ldots, x_{m}, x_{m+1}\right)=\left(f\left(x_{1}, \ldots, x_{m}\right), x_{m+1}\right) .
$$

Suppose that the class of singularities $\Sigma$ is stable under suspension. By this we mean that it is a member $\Sigma_{0}=\Sigma$ of a family

$$
\left\{\Sigma_{r} \subset \mathcal{J}(m+r, n+r)\right\}_{r \geq 0}
$$

such that

$$
\Sigma_{r+1} \cap \mathcal{J}(m+r, n+r)=\Sigma_{r}
$$

and

$$
\mathcal{T}^{\Sigma_{r+1} \mid H^{\bullet}\left(B G L_{m+r} \times B G L_{n+r}, \mathbb{Z}\right)}=\mathcal{T}^{\Sigma_{r}} .
$$

This means that if we specialize

$$
c_{m+r+1}=c_{n+r+1}^{\prime}=0
$$

in the polynomial $\mathcal{T}^{\Sigma_{r+1}}$, we obtain the polynomial $\mathcal{T}^{\Sigma_{r}}$. If the class $\Sigma$ is stable under V-equivalence (cf. $[1, \S I .6 .5]$ ), then it is stable in our sense.

The theorem of Thom has the following refinement due to Damon [4] for a class of singularities $\Sigma$ which is stable under suspension: $\mathcal{T}^{\Sigma}$ is supersymmetric, i.e. it is a polynomial in

$$
\left[\left(1+c_{1}+\cdots+c_{m}\right) /\left(1+c_{1}^{\prime}+\cdots+c_{n}^{\prime}\right)\right]_{i} \quad \text { where } i=1,2, \ldots .
$$

In other words, for a general map $f: M \rightarrow N$,

$$
\mathcal{T}^{\Sigma}\left(c_{1}(M), \ldots, c_{m}(M), c_{1}(N), \ldots, c_{n}(N)\right)
$$

is a polynomial in

$$
c_{i}\left(T M-f^{*} T N\right)=\left[c(T M) / c\left(f^{*} T N\right)\right]_{i} \quad \text { where } i=1,2, \ldots
$$

(here, $T M-f^{*} T N$ is a virtual bundle). Cf. also [9, Theorem 2]. 
3. Schur function expansions. Given a partition $I=\left(i_{1}, \ldots, i_{l}\right) \in \mathbb{N}^{l}$, where $0 \leq i_{1} \leq \cdots \leq i_{l}$, and vector bundles $E$ and $F$ on some variety $X$, the Schur function $\left({ }^{5}\right) S_{I}(E-F)$ is defined by the following determinant:

$$
S_{I}(E-F)=\left|S_{i_{p}+p-q}(E-F)\right|_{1 \leq p, q \leq l},
$$

where the entries are defined by the expression

$$
\sum S_{i}(E-F)=\prod_{b}(1-b) / \prod_{a}(1-a) .
$$

Here, the $a$ 's and $b$ 's are the Chern roots of $E$ and $F$ and the LHS of (3) is the Segre class of the virtual bundle $E-F$. Thus the Schur functions $S_{I}(E-F)$ lie in a ring containing the Chern classes of $E$ and $F$; e.g., we can take the cohomology ring $H^{\bullet}(X, \mathbb{Z})$ or the Chow ring $A(X)$ for a smooth, algebraic $X$. More generally, the Schur functions $S_{I}(-)$ are well defined on the Grothendieck group $K_{0}(X)$ of vector bundles. In particular, given a vector bundle $E$ and a partition $I$, we shall write respectively

$$
S_{I}(E) \text { and } S_{I}(-E)
$$

for $S_{I}(E-0)$ and $S_{I}(0-E)$, where 0 is the zero vector bundle.

We have, for any partition $I$,

$$
S_{I}\left(E^{*}-F^{*}\right)=S_{I^{\sim}}(F-E),
$$

where $I^{\sim}$ denotes the conjugate partition of $I$. In particular,

$$
S_{i}\left(E^{*}-F^{*}\right)=c_{i}(F-E)
$$

for any $i$, so that the Thom polynomial can be equivalently expressed as a polynomial in the

$$
S_{i}\left(R_{m}^{*}-R_{n}^{*}\right) \text { 's. }
$$

Or, evaluated at the Chern classes $c_{i}(M), c_{j}(N)$ of the manifolds involved in the map $f: M \rightarrow N$, it is expressed as a polynomial in the

$$
S_{i}\left(T M^{*}-T N^{*}\right) \text { 's. }
$$

This convention was used in [13], [14], and is used in the present paper.

We refer to [11] and [15] for the theory of Schur functions (a brief account of Schur functions applied to Thom polynomials can be found in [13, Sect. 3] or [14, Sect. 3]). In our notation for partitions we indicate the number of times each integer occurs as a part. For example, we shall denote the partition $(1,2,2,3)$, by $12^{2} 3$.

Using the theory of supersymmetric functions (cf., e.g., [15]), the ThomDamon theorem can be rephrased by saying that there exist $\alpha_{I} \in \mathbb{Z}$ such

$\left({ }^{5}\right)$ Usually this family of functions is called "super Schur functions" or "Schur functions in difference of bundles"; the classical Schur functions $\mathcal{S}_{I}$ will be used in Theorem 3 in the next section. 
that

$$
\mathcal{T}^{\Sigma}=\sum_{I} \alpha_{I} S_{I}\left(R_{m}^{*}-R_{n}^{*}\right),
$$

where the sum is over partitions $I$ with $|I|=\operatorname{codim}(\Sigma)$. The expression in (5) is unique (loc.cit.).

ExAmPLE 1. Let $S_{I}=S_{I}\left(R_{m}^{*}-R_{n}^{*}\right)$. We have the following three formulas valid for $m-n=0,1,2$, respectively:

$$
\begin{aligned}
\mathcal{T}^{A_{4}} & =24 S_{4}+26 S_{13}+10 S_{2^{2}}+9 S_{1^{2} 2}+S_{1^{4}}, \\
\mathcal{T}^{I I I_{2,3}} & =8 S_{35}+4 S_{134}+2 S_{23^{2}} \\
\mathcal{T}^{I_{2,2}} & =S_{24^{2}}+3 S_{145}+7 S_{46}+3 S_{5^{2}} .
\end{aligned}
$$

Note that all the coefficients in the formulas of Example 1 are nonnegative. For a more extensive list of examples, also for maps $\mathbb{C}^{2} \rightarrow \mathbb{C}$, see the last section.

4. Cone classes for ample vector bundles. In the proof of our main result, we shall use the following two results of Fulton and Lazarsfeld from [7]. Recall first some classical definitions and facts from [6] (we shall also follow the notation from that book). Let $E$ be a vector bundle of rank $e$ on $X$. By a cone in $E$ we mean a subvariety of $E$ which is stable under the natural $\mathbb{G}_{\mathrm{m}}$-action on $E$. If $C \subset E$ is a cone of pure dimension $d$, then one may intersect its cycle $[C]$ with the zero-section of the vector bundle:

$$
z(C, E)=s_{E}^{*}([C]) \in A_{d-e}(X),
$$

where $s_{E}^{*}: A_{d}(E) \rightarrow A_{d-e}(X)$ is the Gysin map determined by the zero section $X \rightarrow E$. For a projective variety $X$, there is a well defined degree $\int_{X}: A_{0}(X) \rightarrow \mathbb{Z}$. We first recall the key technical result of [7].

ThEOREM 2 ([7, Theorem 2.1]). Let $E$ be an ample vector bundle of rank $e$ on a projective variety $X$, and let $C \subset E$ be a cone of pure dimension $e$. Then

$$
\int_{X} z(C, E)>0 .
$$

Under the assumptions of the theorem, we also have in $H_{0}(X, \mathbb{Z})$ the homology analog of $z(C, E)$, denoted by the same symbol, and the homology degree $\operatorname{map} \operatorname{deg}_{X}: H_{0}(X, \mathbb{Z}) \rightarrow \mathbb{Z}$. They are compatible with their Chow group counterparts via the cycle map $A_{0}(X) \rightarrow H_{0}(X, \mathbb{Z})(\mathrm{cf}$. [6, Chap. 19]). Thus we have

$$
\operatorname{deg}_{X}(z(C, E))>0 .
$$


Let $P$ be a symmetric polynomial in $e$ variables and of degree $n$. It has a unique presentation as a $\mathbb{Z}$-linear combination

$$
\sum_{I} \beta_{I} \mathcal{S}_{I}
$$

where $|I|=n$, and $\mathcal{S}_{I}$ is the classical Schur function (cf. [11]). Recall that $\mathcal{S}_{I}$ is defined, e.g., by the $e \times e$ determinant (2) with $a$ 's replaced by the given variables, and all $b$ 's equal to zero. For any vector bundle $E$ of rank $e$, we define $P(E)$ to be the image of $P$ under the homomorphism which sends the variables to the Chern roots of $E$. In other words,

$$
P(E)=\sum_{I} \beta_{I} S_{I}(E) .
$$

One says that $P$ is numerically positive for ample vector bundles if for every projective variety $X$ of dimension $n$, and every ample vector bundle $E$ of rank $e$ on $X$

$$
\int_{X} P(E)
$$

is strictly positive. The second result characterizes polynomials numerically positive for ample vector bundles, with the help of Schur functions.

Theorem 3 ([7, Theorem I]). A homogeneous polynomial

$$
\sum_{I} \beta_{I} \mathcal{S}_{I}
$$

where $\beta_{I} \in \mathbb{Z}$, is numerically positive for ample vector bundles iff for any partition $I$ we have $\beta_{I} \geq 0$, and additionally $\sum_{I} \beta_{I}>0$.

For example, the $n$th Chern class $\mathcal{S}_{1^{n}}$ is numerically positive provided $n \leq e$, and in the surface case, i.e. for $n=2$, any polynomial numerically positive for ample vector bundles is either a positive integer multiple of $\mathcal{S}_{1}$, or a $\mathbb{Z}$-linear combination

$$
a \mathcal{S}_{2}+b \mathcal{S}_{1^{2}}
$$

where $a, b \geq 0$ and $a+b>0$ (these results are due respectively to BlochGieseker [3] and Kleiman [10]).

5. Main result. We start with some preliminaries that are known in topology in a much more general framework. We use the notation from Section 2. We first pull back the bundle $\widetilde{\mathcal{J}}$ from $B G$ to $B G L_{m} \times B G L_{n}$ via the map induced by the embedding

$$
G L_{m} \times G L_{n} \hookrightarrow \mathrm{Aut}_{m} \times \mathrm{Aut}_{n} .
$$

Since $G L_{m} \times G L_{n}$ acts linearly on $\mathcal{J}$, the resulting pullback bundle is now the vector bundle on $B G L_{m} \times B G L_{n}$ associated with the representation of 
$G l_{m} \times G L_{n}$ on $\mathcal{J}:$

$$
\mathcal{J}\left(R_{m}, R_{n}\right):=\left(\bigoplus_{i=1}^{k} \operatorname{Sym}^{i}\left(R_{m}^{*}\right)\right) \otimes R_{n} .
$$

The bundle $\mathcal{J}\left(R_{m}, R_{n}\right)$ contains the preimage of $\widetilde{\Sigma} \subset \widetilde{\mathcal{J}}$, denoted by $\Sigma\left(R_{m}, R_{n}\right)$, whose dual class is given by the RHS of (5).

Consider, more generally, a pair of vector bundles $E$ and $F$ of ranks $m$ and $n$ on a variety $X$. We define the following vector bundle on $X$ :

$$
\mathcal{J}(E, F)=\left(\bigoplus_{i=1}^{k} \operatorname{Sym}^{i}\left(E^{*}\right)\right) \otimes F .
$$

In fact, the pair of bundles $(E, F)$ corresponds to a principal $G L_{m} \times G L_{n^{-}}$ bundle $B(E, F)$ and

$$
\mathcal{J}(E, F)=B(E, F) \times_{G L_{m} \times G L_{n}} \mathcal{J}
$$

is the bundle associated with the representation. Similarly, we define the singularity set

$$
\Sigma(E, F)=B(E, F) \times_{G L_{m} \times G L_{n}} \Sigma \subset \mathcal{J}(E, F) .
$$

The dual class $\left({ }^{6}\right)$ of $[\Sigma(E, F)]$ in

$$
H^{2 \operatorname{codim}(\Sigma)}(\mathcal{J}(E, F), \mathbb{Z}) \cong H^{2 \operatorname{codim}(\Sigma)}(X, \mathbb{Z})
$$

is equal to

$$
\sum_{I} \alpha_{I} S_{I}\left(E^{*}-F^{*}\right)
$$

where the $\alpha_{I}$ 's were defined in (5). The argument for that is fairly standard but one has to pass to topological homotopy theory, where each pair of bundles can be pulled back from the universal pair $\left(R_{m}, R_{n}\right)$ of bundles on $B G L_{m} \times B G L_{n}$. It is possible to work entirely with the algebraic varieties. One can use the Totaro construction and representability for affine varieties ([18, proof of Theorem 1.3]).

The main result of the present paper, suggested/conjectured in [13], [14], and in [5] for Thom-Boardman singularities, is

TheOREM 4. Let $\Sigma$ be a stable, nontrivial class of singularities. Then the Thom polynomial $\mathcal{T}^{\Sigma}$ is nonzero, and for any partition I the coefficient $\alpha_{I}$ in the Schur function expansion of $\mathcal{T}^{\Sigma}(c f .(5))$ is nonnegative.

Proof. We follow the notation from the first part of this section. Let $c=\operatorname{codim}(\Sigma)$ (so that for any partition $I$ with $\alpha_{I} \neq 0$, we have $|I|=c$ ). Suppose that $X$ is a projective variety of dimension $c, F$ is an ample vector

$\left({ }^{6}\right)$ A precise definition of the dual class of $[\Sigma(E, F)]$, in the case when $X$ is singular, is given in Note 6 . 
bundle on $X$ with $\operatorname{rank}(F)=n^{\prime}=n+r \geq c$, and $E=\mathbf{1}^{m^{\prime}}$ is a trivial bundle on $X$ of rank $m^{\prime}=m+r$ (for some $r \geq 0$ ).

The variety $\Sigma\left(\mathbf{1}^{m^{\prime}}, F\right)$ is a cone in $\bar{J}\left(\mathbf{1}^{m^{\prime}}, F\right)$ because $\mathbb{G}_{\mathrm{m}} \subset$ Aut $_{n^{\prime}}$. From the first part of this section, we know that the cone class

$$
z(\Sigma(E, F), \mathcal{J}(E, F)) \in H_{0}(X, \mathbb{Z})
$$

is dual to the universal expression (8):

$$
\sum_{I} \alpha_{I} S_{I}\left(E^{*}-F^{*}\right)=\sum_{I} \alpha_{I} S_{I}\left(-F^{*}\right)=\sum_{I} \alpha_{I} S_{I \sim}(F)
$$

(we use here (2) and (4)). Applying the notation of Theorem 3, we set

$$
P=\sum_{I} \alpha_{I} \mathcal{S}_{I^{\sim}}
$$

Since a direct sum of ample vector bundles is ample [8, Proposition 2.2], the vector bundle

$$
\mathcal{J}\left(\mathbf{1}^{m^{\prime}}, F\right)=F^{\oplus N}
$$

(for some integer $N$ ) is ample. Hence, we have, by the inequality (7),

$$
\int_{X} P(F)=\operatorname{deg}_{X}\left(z\left(\Sigma\left(\mathbf{1}^{m^{\prime}}, F\right), F^{\oplus N}\right)\right)>0
$$

that is, the polynomial $P$ is numerically positive for ample vector bundles. We conclude, by Theorem 3, that the coefficients $\alpha_{I}$ in (5) are nonnegative. Moreover, we have $\sum_{I} \alpha_{I}>0$, which implies that $\mathcal{T}^{\Sigma} \neq 0$.

Theorems 2 and 3 are valid over any algebraically closed field of arbitrary characteristic (cf. [7]). Therefore, our main result would hold in that generality provided that one develops a suitable theory of Thom polynomials. Of course, usual cohomology should be replaced by Chow rings (cf. [18]). By Theorem 1.3 (loc.cit.), the theory of characteristic classes is reduced to calculations in the Chow ring of a classifying space.

REMARK 5 . The functor of $k$-jets

$$
E, F \mapsto \mathcal{J}(E, F)=\left(\bigoplus_{i=1}^{k} \operatorname{Sym}^{i}\left(E^{*}\right)\right) \otimes F
$$

and the cone $\Sigma(E, F)$ can be replaced by a more general construction: an arbitrary functor $\phi(E, F)$ and a cone bundle $\Sigma(E, F) \subset \phi(E, F)$. We assume the following two properties:

1. the class $z(\Sigma(E, F), \phi(E, F))$ is stable under simultaneous addition of the same bundle to $E$ and $F$;

2. the assignment $F \mapsto \phi\left(\mathbf{1}^{m}, F\right)$ (or $E \mapsto \phi\left(E^{*}, \mathbf{1}^{n}\right)$ ) preserves ampleness.

Examples of functors preserving ampleness for fields of characteristic zero are polynomial functors. They are, at the same time, quotient functors and 
subfunctors of tensor power functors (cf. [8]). The analog of Theorem 4, asserting the nonnegativity of the coefficients in the expansion of the dual class of $[\Sigma(E, F)]$ in the basis of the $S_{I}(E-F)$ 's, remains then valid.

Note 6 . We give here a precise definition of the dual class of $[\Sigma(E, F)]$ for possibly singular $X$. This class will lie in $H^{2 c}(X, \mathbb{Z})$ (recall that $c=$ $\operatorname{codim}(\Sigma)$ ). Let $Y \subset \Sigma$ be the set of singular points of $\Sigma$, i.e.

$$
\Sigma \backslash Y \subset \mathcal{J} \backslash Y
$$

is a submanifold. The set $Y$ is $G L_{m} \times G L_{n}$-invariant. For a pair $E, F$ of bundles we construct the fibering

$$
Y(E, F)=B(E, F) \times_{G L_{m} \times G L_{n}} Y \subset \Sigma(E, F) \subset \mathcal{J}(E, F)
$$

with fiber $Y$. We have $\operatorname{codim}_{\mathbb{C}} Y \geq c+1$, and therefore

$$
H^{i}(\mathcal{J} \backslash Y, \mathbb{Z}) \cong H^{i}(\mathcal{J}, \mathbb{Z})
$$

for $i \leq 2 c$. Hence

$$
H^{i}(\mathcal{J}(E, F) \backslash Y(E, F), \mathbb{Z}) \cong H^{i}(\mathcal{J}(E, F), \mathbb{Z})
$$

in the same range of degrees, and it is enough to define the desired class in $H^{2 c}(\mathcal{J}(E, F) \backslash Y(E, F), \mathbb{Z})$. Note that $\Sigma(E, F) \backslash Y(E, F)$ has a normal bundle. Its Thom class defines an element in

$$
H^{2 c}(\mathcal{J}(E, F) \backslash Y(E, F), \mathcal{J}(E, F) \backslash \Sigma(E, F) ; \mathbb{Z}) .
$$

The image of this element in

$$
H^{2 c}(\mathcal{J}(E, F) \backslash Y(E, F), \mathbb{Z}) \cong H^{2 c}(\mathcal{J}(E, F), \mathbb{Z}) \cong H^{2 c}(X, \mathbb{Z})
$$

is the desired class. Verification that this class is natural with respect to pull back of vector bundles is left to the reader.

6. Examples. Let $S_{I}=S_{I}\left(R_{m}^{*}-R_{n}^{*}\right)$ in the notation of Section 2 . We list several examples of singularities $\Sigma[n-m]: M \rightarrow N$, where $m=$ $\operatorname{dim}(M) \leq n=\operatorname{dim}(N)$. All of them were computed by the "method of restriction equations" of [16]. In (a) below, we give the Schur function expansions of Thom polynomials for singularities $\Sigma[0]$ with codimension $\leq 6$ from [16, p. 508]. In (b), we give the Schur function expansion of the Thom polynomials for the singularities $\Sigma[1]$ from [16, p. 512]. In (c), we list some examples from [13], [14]. (Note that in [13] and [14] we used the notation $\eta(n-m+1)$ for a singularity $\eta:\left(\mathbb{C}^{m}, 0\right) \rightarrow\left(\mathbb{C}^{n}, 0\right)$.)

(a)

$$
\begin{aligned}
A_{1}[0]: & S_{1} \\
A_{2}[0]: & 2 S_{2}+S_{1^{2}} \\
A_{3}[0]: & 6 S_{3}+5 S_{12}+S_{1^{3}} \\
A_{4}[0]: & 24 S_{4}+26 S_{13}+10 S_{2^{2}}+9 S_{1^{2} 2}+S_{1^{4}} \\
I_{2,2}[0]: & S_{2^{2}} \\
A_{5}[0]: & 120 S_{5}+154 S_{14}+92 S_{23}+71 S_{1^{2} 3}+14 S_{1^{3} 2}+35 S_{12^{2}}+S_{1^{5}}
\end{aligned}
$$




$$
\begin{aligned}
I_{2,3}[0]: & 4 S_{23}+2 S_{12^{2}} \\
A_{6}[0]: & 720 S_{6}+1044 S_{15}+770 S_{24}+266 S_{3^{2}}+580 S_{1^{2} 4}+455 S_{123}+70 S_{2^{3}}+ \\
& 155 S_{1^{3} 3}+84 S_{1^{2} 2^{2}}+20 S_{1^{4} 2}+S_{1^{6}} \\
I_{2,4}[0]: & 16 S_{24}+4 S_{3^{2}}+12 S_{123}+5 S_{2^{3}}+2 S_{1^{2} 2^{2}} \\
I_{3,3}[0]: & 2 S_{24}+6 S_{3^{2}}+3 S_{123}+S_{1^{2} 2^{2}}
\end{aligned}
$$

(b)

$$
A_{1}[1]: S_{2}
$$

$A_{2}[1]: 4 S_{4}+2 S_{13}+S_{2^{2}}$

$A_{3}[1]: 36 S_{6}+30 S_{15}+19 S_{24}+5 S_{3^{2}}+6 S_{1^{2} 4}+5 S_{123}+S_{2^{3}}$

$A_{4}[1]: 507 S_{8}+555 S_{17}+391 S_{26}+240 S_{35}+76 S_{4^{2}}+216 S_{1^{2} 6}+210 S_{125}+$

$I I I_{2,2}[1]: S_{3^{2}}$

$$
104 S_{134}+55 S_{2^{2} 4}+21 S_{23^{2}}+24 S_{1^{3} 5}+26 S_{1^{2} 24}+10 S_{1^{2} 3^{2}}+9 S_{12^{2} 3}+S_{2^{4}}
$$

$I_{2,2}[1]: 3 S_{34}+S_{13^{2}}$

$I I I_{2,3}[1]: 8 S_{35}+4 S_{134}+2 S_{23^{2}}$

(c)

$I_{2,2}[1]: S_{13^{2}}+3 S_{34}$

$I_{2,2}[2]: S_{24^{2}}+3 S_{145}+7 S_{46}+3 S_{5^{2}}$

$I_{2,2}[3]: S_{35^{2}}+3 S_{256}+7 S_{157}+3 S_{16^{2}}+15 S_{58}+10 S_{67}$

Finally, we list the Thom polynomials for the functions $\mathbb{C}^{2} \rightarrow \mathbb{C}$ of the singularities $A, D, E$ with codimension at most 6 , computed by the "method of restriction equations" of [16]. The results agree with those in [9] given in the basis of monomials in Chern classes.

$$
\begin{aligned}
A_{1}: & S_{1^{2}} \\
A_{2}: & 2 S_{1^{3}}+2 S_{12} \\
A_{3}: & 5 S_{1^{4}}+11 S_{1^{2} 2}+6 S_{2^{2}}+6 S_{13} \\
A_{4}: & 12 S_{1^{5}}+44 S_{1^{3} 2}+44 S_{12^{2}}+56 S_{1^{2} 3}+36 S_{23}+24 S_{14} \\
D_{4}: & S_{1^{5}}+3 S_{1^{3} 2}+6 S_{12^{2}}+2 S_{1^{2} 3}+4 S_{23} \\
A_{5}: & 30 S_{1^{6}}+160 S_{1^{4} 2}+248 S_{1^{2} 2^{2}}+338 S_{1^{3} 3}+434 S_{123}+328 S_{1^{2} 4}+108 S_{3^{2}}+ \\
& 228 S_{24}+120 S_{15} \\
D_{5}: & 4 S_{1^{6}}+18 S_{142}+42 S_{1^{2} 2^{2}}+26 S_{1^{3} 3}+64 S_{123}+12 S_{1^{2} 4}+24 S_{3^{2}}+24 S_{24} \\
A_{6}: & 79 S_{1^{7}}+566 S_{1^{5} 2}+1238 S_{1^{3} 2^{2}}+1723 S_{1^{4} 3}+3473 S_{1^{2} 23}+2736 S_{1^{3}}+1834 S_{13^{2}} \\
& +3898 S_{124}+2220 S_{1^{2} 5}+1260 S_{34}+1632 S_{25}+720 S_{16} \\
D_{6}: & 8 S_{1^{7}}+50 S_{1^{5} 2}+138 S_{1^{3} 2^{2}}+118 S_{1^{4} 3}+348 S_{1^{2} 23}+124 S_{1^{3} 4}+224 S_{13^{2}}+ \\
& 320 S_{124}+48 S_{1^{25}}+144 S_{34}+96 S_{25} \\
E_{6}: & 3 S_{1^{7}}+18 S_{1^{5} 2}+54 S_{1^{3} 2^{2}}+39 S_{1^{4} 3}+129 S_{1^{2} 23}+36 S_{1^{3} 4}+102 S_{13^{2}}+102 S_{124}+ \\
& 12 S_{1^{2} 5}+60 S_{34}+24 S_{25}
\end{aligned}
$$

Acknowledgments. The first author thanks Alain Lascoux for helpful discussions on Schur functions and Thom polynomials. The second author thanks Maxim Kazarian for suggesting Remark 5. Both authors thank Özer Öztürk for his help with computer computations. 


\section{References}

[1] V. I. Arnol'd, S. M. Gusel̆n-Zade and A. N. Varchenko, Singularities of Differentiable Maps, I, Monogr. Math. 82, Birkhäuser, 1985.

[2] V. I. Arnol'd, V. A. Vasil'ev, V. V. Goryunov and O. V. Lyashko, Singularities. Local and global theory, Encyclopaedia Math. Sci. 6 (Dynamical Systems VI), Springer, 1993.

[3] S. Bloch and D. Gieseker, The positivity of the Chern classes of an ample vector bundle, Invent. Math. 12 (1971), 112-117.

[4] J. Damon, Thom polynomials for contact singularities, Ph.D. Thesis, Harvard, 1972.

[5] L. Fehér and B. Kömüves, On second order Thom-Boardman singularities, Fund. Math. 191 (2006), 249-264.

[6] W. Fulton, Intersection Theory, Springer, 1984, 1998.

[7] W. Fulton and R. Lazarsfeld, Positive polynomials for ample vector bundles, Ann. of Math. 118 (1983), 35-60.

[8] R. Hartshorne, Ample vector bundles, Publ. Math. IHES 29 (1966), 63-94.

[9] M. E. Kazarian, Classifying spaces of singularities and Thom polynomials, in: New Developments in Singularity Theory, NATO Sci. Ser. II Math. Phys. Chem. 21, Kluwer, 2001, 117-134.

[10] S. Kleiman, Ample vector bundles on algebraic surfaces, Proc. Amer. Math. Soc. 21 (1969), 673-676.

[11] A. Lascoux, Symmetric Functions and Combinatorial Operators on Polynomials, CBMS Reg. Conf. Ser. Math. 99, Amer. Math. Soc., 2003.

[12] Ö. Öztürk, On Thom polynomials for $A_{4}(-)$ via Schur functions, Serdica Math. J. 33 (2007), to appear.

[13] P. Pragacz, Thom polynomials and Schur functions I, math.AG/0509234.

[14] - Thom polynomials and Schur functions: the singularities $I_{2,2}(-)$, Ann. Inst. Fourier (Grenoble) 57 (2007), to appear.

[15] P. Pragacz and A. Thorup, On a Jacobi-Trudi identity for supersymmetric polynomials, Adv. Math. 95 (1992), 8-17.

[16] R. Rimányi, Thom polynomials, symmetries and incidences of singularities, Invent Math. 143 (2001), 499-521.

[17] R. Thom, Les singularités des applications différentiables, Ann. Inst. Fourier (Grenoble) 6 (1955-56), 43-87.

[18] B. Totaro, The Chow ring of a classifying space in: Algebraic K-theory, W. Raskind et al. (eds.), Proc. Sympos. Pure Math. 67, Amer. Math. Soc., 1999, 249-281.

[19] S. Veigneau, ACE, an algebraic combinatorics environment for the computer algebra system MAPLE, 1998.

Institute of Mathematics

Polish Academy of Sciences

Śniadeckich 8

00-956 Warszawa, Poland

E-mail: P.Pragacz@impan.gov.pl
Department of Mathematics

Warsaw University

Banacha 2

02-097 Warszawa, Poland

E-mail: aweber@mimuw.edu.pl 